
matérielle de l'éducation

\title{
Le fonds des films fixes du Cedrhe : illustration de l'histoire d'un support pédagogique
}

\section{Sylvain Wagnon and Hélène André}

\section{(2) OpenEdition}

\section{Journals}

Electronic version

URL: http://journals.openedition.org/trema/3117

DOI: $10.4000 /$ trema.3117

ISSN: 2107-0997

\section{Publisher}

Faculté d'Éducation de l'université de Montpellier

\section{Printed version}

Date of publication: 1 June 2014

Number of pages: 8 - 23

ISBN: 1167-315X

ISSN: $1167-315 X$

Electronic reference

Sylvain Wagnon and Hélène André, "Le fonds des films fixes du Cedrhe : illustration de l'histoire d'un support pédagogique », Tréma [Online], 41 | 2014, Online since 01 June 2016, connection on 19 April 2019. URL : http://journals.openedition.org/trema/3117 ; DOI : 10.4000/trema.3117

This text was automatically generated on 19 April 2019.

Trema 


\title{
Le fonds des films fixes du Cedrhe: illustration de l'histoire d'un support pédagogique
}

\author{
Sylvain Wagnon and Hélène André
}

1 Objet patrimonial depuis l'arrivée de la diapositive dans les années soixante et des supports informatiques à partir des années 80 , le film fixe, par ses limites chronologiques circonscrites, offre la possibilité d'une mise en perspective de nombreux débats actuels concernant l'éducation aux médias et les enjeux de la mise en place d'un nouveau support pédagogique dans l'enseignement. Notre étude s'appuie sur le fonds de films fixes du Cedrhe pour aborder l'histoire de cet outil pédagogique. Car au même titre que les textes officiels ou les manuels scolaires, avec leurs propres caractéristiques, les films fixes permettent de mettre l'accent sur une nécessaire histoire des pratiques pédagogiques afin d'esquisser une réflexion sur ce que l'on enseigne et comment on l'enseigne.

Nous voudrions, en nous appuyant sur l'analyse du fonds des films fixes du Cedrhe, proposer une réflexion autour de trois axes $^{1}$. Dans une première partie, nous souhaiterions faire connaitre le fonds des films fixes du Cedrhe, l'un des plus importants en France. L'analyse sommaire de ce fonds permet à la fois d'établir une typologie du film fixe mais aussi d'engager une première réflexion sur ses usages dans les classes.

3 Dans une seconde partie, nous analyserons, à travers l'histoire du film fixe depuis les années 20 , en quoi il fut, avant d'être un support d'enseignement de l'école, un outil au service de l'enseignement religieux et des activités « périscolaires ».

4 Enfin dans une troisième partie, à partir de l'analyse du fonds des films fixes du Cedrhe mais aussi des revues pédagogiques des années 30 aux années 60 , nous analyserons le film fixe en tant qu'enjeu pédagogique. À cet égard, l'analyse de plusieurs revues professionnelles nous permettra d'esquisser une périodisation et des éléments d'analyse de l'histoire du film fixe dans l'enseignement français qui reste liée au débat pédagogique de l'usage de l'image dans l'enseignement. En effet, perçu dès les années 30 par les chercheurs et une partie des enseignants comme un symbole des « méthodes actives », il 
fut aussi observé avec méfiance par ceux qui craignaient que ce nouveau médium fragilise le rôle de l'enseignant.

\section{Les films fixes du Cedrhe: un fonds à découvrir}

5 Un premier inventaire, nous a permis de comptabiliser plus de 4000 films fixes, avec presque autant de doublons, qui commencent à être catalogués dans une banque de données. Ainsi, le fonds de films fixes du Cedrhe apparaît comme l'un des plus importants en France avec ceux du CREDIE de l'ESPE de Lyon (10000 films fixes) et celui de l'Association pour la sauvegarde des films fixes du diocèse d'Anjou (ASFFA, 3000 films).

6 Les films fixes sont muets et de formats multiples ( $8 \mathrm{~mm}, 9.5 \mathrm{~mm}, 16 \mathrm{~mm}$ et $35 \mathrm{~mm}$ ). Ils ont l'aspect d'une pellicule, transparente ou noire, photographique avec ses bords crantés et se composent de vues positives en noir et blanc, parfois avec de la couleur suivant les périodes. Les films peuvent être composés de dessins, de photographies, de cartes et sont accompagnés la plupart du temps d'un commentaire très bref. Les films sont roulés dans de petites boîtes carrées ou rondes, en carton, métal ou plastique.

7 La «durée » du film fixe est tout à fait variable, d'une dizaine de vues à près d'une centaine de vues. Mais les films $35 \mathrm{~mm}$ à double perforation sont d'une part les plus longs et d'autre part ceux qui furent les plus nombreux et les plus utilisés.

Par l'aspect des boîtes, on peut même facilement identifier quelques éditeurs : les Éditions filmées avec ses boîtes métalliques ou les éditions Larousse qui se distinguent par des boites rondes en cartons, ornées de pastilles de couleurs selon la discipline, et de dimension légèrement supérieure au film, afin de pouvoir contenir une notice explicative. A priori, tous les films scolaires sont accompagnés de notices, même si nous ne les avons pas retrouvées dans les boîtes. Ces notices explicatives constituent un support avec quelques commentaires, qui proposent, en quelque sorte, un cours " prêt à l'emploi » pour l'enseignant avec une explication des différentes vues et «l'articulation » qui en découle.

9 Le Cedrhe, bibliothèque de recherche en histoire de l'éducation, a été fondé par Pierre Guibbert en 1992, enseignant dans le secondaire, puis enseignant-chercheur à l'IUFM de Montpellier. Pierre Guibbert fut un passionné de cinéma. Dans les années 1960, il rejoint Marcel Oms, fondateur de la cinémathèque de Toulouse et de l'Institut Jean Vigo, et participe activement à la création et à la diffusion de plusieurs publications (Cahiers de la cinémathèque, revue Archives, festival Confrontation) ${ }^{2}$.

Les dons, dépôts et échanges avec la cinémathèque de Toulouse expliquent la présence d'un fonds de films religieux à côté des films d'enseignement issus des écoles primaires de l'Hérault.

11 Ensuite, Pierre Guibbert fut l'auteur d'une thèse sur les circonstances, modalités et significations de l'œuvre d'Eisenstein (1972) ${ }^{3}$, ce qui explique en grande partie que le Cedrhe possède un fonds de films soviétiques.

12 Un premier inventaire nous a permis de comprendre l'importance de ce fonds par sa quantité mais aussi par sa variété tant du point de vue chronologique (films des années 30 à 70) que du point de vue thématique (sciences, géographie, histoire, religion, hygiène, contes, vie quotidienne...) ${ }^{4}$. 
13 Nous avons, dans un premier temps, repris la typologie large et classique des films fixes (religieux, récréatifs et pédagogiques). Une typologie plus fine par discipline est apparue très aléatoire. Pour faciliter la recherche, nous avons établi une conservation par maisons d'éditions. En effet, il est apparu que de nombreux films " dépassaient » une discipline scolaire en tant que telle et pouvait être rangée dans plusieurs thématiques rendant inopérantes une recherche. L'intégration dans le SUDOC et la constitution d'une base de données avec des mots clés permettront, en outre, d'établir des recherches croisées.

Ensuite, la plupart de ces films s'adresse à l'enseignement primaire. Si l'histoire, la géographie et les sciences semblent dominantes pour les éditions scolaires (Larousse, Éditions filmées, OSEF Office supérieur de l'enseignement par les films...), on voit également apparaître de nombreux films ayant trait à la vie quotidienne (le geste professionnel, la prévention contre l'alcool, l'épargne...).

15 Tout en ayant une histoire commune avec le cinéma éducatif, le film fixe, a un itinéraire singulier. En effet, il fut d'abord un enjeu idéologique entre catholiques et laïcs avant d'être un enjeu pédagogique au sein de l'école.

\section{Film animé et film fixe : une histoire initiale commune?}

16 Les débuts de l'histoire du film fixe semblent se conjuguer avec celui du cinéma animé éducatif. Avant la première guerre mondiale, seuls quelques pionniers s'intéressent à l'introduction du film dans l'enseignement. Ainsi le producteur et réalisateur Jean Benoît Levy (1888-1959) propose dès 1909 "l'application du cinéma à l'instruction », en intervenant dans les congrès de la Ligue de l'enseignement et des mutualités scolaires pour encourager les instituteurs à adopter « le nouvel instrument de travail scolaire ${ }^{6}$ ».

17 Parallèlement, les premières volontés politiques apparaissent clairement à l'issue du premier conflit mondial. Avec la création d'une commission extraparlementaire «chargée de rechercher les meilleurs moyens de généraliser l'utilisation cinématographique dans les différentes branches de l'enseignement ». Le rapport de la commission note la nécessité d'un contrôle accru du contenu des films pour éviter qu'il «corrompe " les enfants mais se déclare favorable à l'usage du film animé et fixe dans l'enseignement à tous les degrés 7 . Ainsi, la circulaire du 20 novembre $1921^{8}$ du ministère de l'Instruction publique décide toute une série de dispositions pour développer le film dans l'école. D'une part, la possibilité d'obtenir par le ministère le paiement d'un tiers des dépenses d'achat et d'installations du cinéma dans une école.

D'autre part, le ministère crée une commission permanente chargée de toutes les questions inhérentes au film : visa, prises de vues, centres régionaux du cinéma, dépôt de films dans les Inspections académiques, régime administratif et contrôle des films, exonération de l'impôt sur les spectacles, liste des appareils agréés, etc.

20 En 1922, le premier congrès national du cinéma éducatif, sous l'égide de la Direction nationale de l'enseignement technique, achève le dispositif gouvernemental créant les conditions d'un essor du cinéma éducatif et la diffusion des films animés et fixes au sein du système éducatif français. 


\section{Le fruit d'une révolution technique}

21

Mais l'essor particulier du film fixe est aussi le fruit d'une innovation technique qui permet une diffusion peu couteuse du film fixe par rapport au film animé. En effet, la société Pathé-Cinéma lance en 1923 un nouvel appareil le Pathé baby, «le cinéma chez soi » qui permet de remplacer la vue sur verre. Très vite cette innovation technique est suivie par la création de toute une série de projecteurs comme le «Mistral» ou «le Provence " marquant l'intérêt d'entreprises privées pour ce nouveau marché. La société Pathé-cinéma souligne ce que sont, selon elle, les avantages du film fixe :

«Le but poursuivi par notre société a été de mettre à la portée de tous, pour un prix modique, un choix de vues sélectionnées avec soin, accompagnées d'un texte explicatif, documenté, et pouvant rendre les mêmes services que les clichés de verre, d'usage général mais de prix élevé et de manipulation délicate en raison de leur fragilité ${ }^{9}$.

Ainsi le film fixe commence à se différencier fortement du film animé qui de son côté, à la même époque, devient parlant; le film fixe permet au maitre de conserver le monopole de la parole par rapport au film.

Mais si le ministère de l'instruction publique se montre favorable à l'usage du cinéma et en particulier du film fixe, ce dernier reste dans l'entre deux-guerres l'apanage de la catéchèse catholique.

\section{Du catéchisme à l'enseignement laïc}

Les premiers films fixes ont été utilisés de façon généralisée par les patronages chrétiens et lors du catéchisme. Édités par milliers, ces films racontant l'histoire sainte sont des supports appréciés par le clergé et les missionnaires ${ }^{10}$. Ils laissent au prêtre la possibilité de commenter, d'expliquer à sa façon sans être contredit.

Ces films fixes religieux, dont le Cedrhe possède près de 600 titres différents, ont été réalisés par plusieurs maisons d'éditions. Par exemple, la Maison de la Bonne Presse qui édite de nombreux films fixes religieux, est aussi la maison d'édition qui possède des journaux chrétiens tels que Le pèlerin et le quotidien La Croix. Dès la création du cinéma en 1895, Georges-Michel Coissac (1868-1946) est chargé par le groupe éditorial religieux d'organiser un réseau de projections d'abord de projections sur verre, puis de films fixes plus malléables et utilisables. Cette diffusion dirigée par «la centrale catholique du cinéma » illustre un encadrement paroissial par les films fixes et fut d'ailleurs un modèle pour le ministère de l'instruction publique, qui charge Georges-Michel Coissac d'un rapport sur la question en $1926^{11}$.

Les milieux catholiques organisent également la revue cinématographique, Le fascinateur, qui doit se placer clairement dans la lutte d'influence avec le camp laïc. En 1904, Constant Pautrier écrit :

«C'est sur tous les terrains que se poursuit aujourd'hui la lutte engagée contre le Christ par les ennemis de l'Église; et de tous les moyens mis en œuvre pour semer l'incrédulité et insuffler la haine chez les générations qui montent, il n'en est pas de plus fréquemment employé que l'enseignement, je dirais même la prédication par les yeux ${ }^{12} »$. 
Cette lutte d'influence illustre que l'usage de l'image dans les apprentissages est devenu une évidence pour tous. La revue pédagogique qui reste une source pour l'enseignement public, insiste ainsi, dès 1899, sur l'importance de l'image dans les conférences pédagogiques que les instituteurs publics doivent élaborer pour l'ensemble de la population :

« Nos éducateurs ont mis à profit, dans l'intérêt de leur cause, les découvertes les plus récentes, même celle du cinématographe. Point de cours d'adultes, point de conférences sérieuses sans projections multipliées ${ }^{13}$ ».

Toujours dans La revue pédagogique en 1920, Auguste Bessou, rapporteur de la commission extra-parlementaire chargée d'étudier les moyens de généraliser l'application du cinématographe dans les différentes branches de l'enseignement ${ }^{14}$, souligne :

«Le cinématographe détruit ces effets de l'ignorance qui se croit avisée parce qu'elle est méfiante et bornée. Il initie l'enfant, dès son plus jeune âge, au mécanisme si complexe de la vie moderne ${ }^{15} »$.

Après la première guerre mondiale, la «riposte laïque » est organisée d'une part, à l'initiative de la Ligue de l'Enseignement qui crée une revue spécifique Ciné-scola ${ }^{16}$, " vade-mecum de l'enseignement par le cinéma ». D'autre part, par la création des offices du cinéma éducateur ${ }^{17}$ dont les premiers datent de 1921. Mais, il faut attendre 1933 avec la fondation de l'Union française des offices du cinéma scolaire et éducateur (UFOCEL) ${ }^{18}$, qui fédère et coordonne les différents offices face au réseau des salles paroissiales. L'UFOCEL structure l'ensemble des offices comme un réseau d'enseignement, d'éducation et de propagande par le cinéma ${ }^{19}$.

Les offices peuvent s'appuyer sur la quantité de films fixes et animés issus de la cinémathèque du Musée pédagogique qui dépend du ministère de l'Instruction publique et des Beaux-arts. Le Code Soleil, le «bréviaire» des instituteurs et institutrices de l'enseignement public précise dans son édition de 1926 :

«Organisons des séances de lectures commentées, de conférences et même si possible, de conférences avec projections fixes ou filmées grâce au cinéma scolaire qui deviendra de plus en plus, le plus précieux vulgarisateur de connaissance jusque dans les communes rurales ".

31 Ainsi l'image, qu'elle soit animée ou fixe, s'intègre dans l'enseignement. L'usage didactique de l'image est utilisé par l'institution scolaire comme un vecteur puissant pour développer une propagande morale et hygiénique ${ }^{20}$.

\section{Un essor continu empreint de méfiance}

Mais si le cinéma animé éducateur et le film fixe d'enseignement connaissent un essor conjoint dans les années 20, plusieurs «freins » expliquent le fait qu'il reste toutefois marginal du point de vue de l'enseignement scolaire.

Tout d'abord du côté des enseignants, le cinéma est considéré pour beaucoup comme une possible interférence entre leur discours et les élèves. Les militants du cinéma éducateur présentent cette crainte d'une «fragilisation » de l'autorité du maître par le cinéma et donnent au cinéma un rôle avant tout illustratif et d'accompagnement. À ce titre, le film fixe apparaît comme un support intermédiaire qui ne remet pas en cause l'autorité du maître. 

Mais cette méfiance, plus qu'une réelle défiance à l'égard de cette «nouvelle technologie » rend aussi difficile un essor économique du cinéma d'enseignement car pour les entreprises cinématographiques, il représente encore un «marché » peu rentable. Les catalogues des années 30 restent très modestes et ne satisfont aucunement les enseignants pionniers en la matière, les formats des films fixes restent multiples et peu compatibles avec tous les projecteurs en usage.

En 1930, la revue Tout Cinéma restait optimiste et précisait :

«Toutefois, il est bien évident que le pas décisif ne sera franchi que si l'État accorde, non seulement son appui moral aux initiatives privées du cinéma d'enseignement mais s'il élabore un programme destiné à être appliqué aux écoles publiques. On pourrait également envisager un vaste développement du cinéma d'éducation dans les œuvres postscolaires ${ }^{21} »$.

Mais en 1931, un accident émeut l'opinion publique et illustre la crainte des dangers techniques du cinéma. Au cours d'une projection cinématographique, le feu se déclare dans la cabine de projection, l'instituteur Stéphane Pitard (1908-1931) arrive à sauver les 200 enfants présents de l'école de garçons de Château-Renault mais trouve la mort des suites de ses brûlures. Après cet épisode, le ministre de l'instruction publique et des Beaux-arts, Mario Roustan, prend plusieurs arrêtes interdisant l'usage de films inflammables dans les écoles et en présence d'écoliers. Toutefois, sur le principe, il encourage officiellement la poursuite de l'usage des films à l'école en faisant savoir à tous les inspecteurs d'académie que «des subventions pourront être accordées sur les crédits du ministère de l'Éducation nationale pour l'acquisition, sous réserve de l'approbation technique, d'appareils de projections ${ }^{22} »$.

En 1945, l'UFOCEL est remplacé par l'Union française des œuvres laïques d'éducation par l'image et par le son (l'UFOLEIS) qui est chargée, en lien avec le ministère de l'éducation nationale, d'organiser l'essor du cinéma à l'école. Mais c'est surtout la circulaire du 30 janvier 1952 dite "loi Barangée ${ }^{23}$ ", qui, en accordant aux établissements scolaires d'importantes subventions pour l'achat de matériel d'enseignement, permit un usage généralisé dans les classes des films fixes. Facile d'utilisation, le film fixe est le favori des achats des établissements scolaires, très loin devant les autres projections cinématographiques ou autres matériels, comme les machines à écrire.

Le préambule de la circulaire du 30 janvier 1952 est tout à fait significatif :

«Le film apporte dans la classe une reproduction des êtres et des choses, rend l'observation possible là où elle est impossible directement et possède une puissance incomparable d'explication et de suggestion, il est par là même un instrument pédagogique admirablement efficace, lorsqu'il est utilisé à bon escient, c'est-à-dire lorsqu'il intervient comme élément d'information dans une classe ${ }^{24}$ ».

\section{2 ou le paradigme idéalisé du film fixe}

La loi Barangé marque la volonté gouvernementale de soutenir activement cette innovation, plus de 16 milliards de francs sont dépensés en 1952-1953 ${ }^{25}$ pour le matériel d'enseignement technique et audiovisuel. Cette loi insuffle un véritable essor économique au film fixe en créant un marché où de nombreux éditeurs vont s'engouffrer. Par exemple, les éditions Larousse présentent un catalogue complet qui, selon leur brochure, 
«offre un choix abondant de films conçus pour tous les niveaux de l'enseignement, pour toutes les matières susceptibles d'être représentées par l'image ${ }^{26} »$.

Puis Larousse souligne l'intérêt pédagogique du film fixe :

«L'expérience a démontré dans le domaine pédagogique la supériorité du film fixe sur le film animé ; il imprègne plus profondément la mémoire de l'enfant, laisse plus de liberté à l'éducateur dans ses explications. Il est moins onéreux et de maniement plus facile».

Enfin, Larousse marque son accompagnement précoce pour tout ce qui est scolaire, ce qui est de bonne guerre face à la concurrence :

«Faut-il rappeler que, dès 1896, la librairie Larousse commençait de fournir aux membres de l'enseignement des vues à caractère pédagogique?

Moderne hier, la librairie Larousse l'est demeurée. Les films dont on trouvera plus loin l'inventaire sont conformes aux programmes actuels, composés d'images neuves, originales, efficaces et faites - comme les verres peints de jadis - pour réconcilier l'étude avec la joie ».

« Nous recommandons tout spécialement pour l'utilisation rationnelle des crédits offerts par loi Barangé, nos films, projecteurs, et les ouvrages de bibliothèques et de complément suivants".

42 La circulaire du 30 janvier 1952 dont l'objet est la production et la distribution des films d'enseignement précise la volonté ministérielle dans ce domaine. Cette circulaire ainsi que celle du 21 février 1952 font suite à la publication de la loi du 28 septembre 1951 dite loi Barangé qui, entre autres, définit les nouvelles ressources dont les communes disposent en faveur des écoles.

Le film est bien défini comme un auxiliaire d'enseignement, sous l'autorité du maître et le ministre de conclure :

«L'œuvre filmée, au même titre que l'œuvre littéraire ou dramatique, en raison

même de l'importance que tient dans la vie moderne ce nouveau mode d'expression

de l'art et de la pensée, est et deviendra de plus en plus un objet d'enseignement ».

Mais le congrès des Écoles maternelles qui se tient à Toulouse du 15 au 17 juillet 1952 nous permet de comprendre aussi toute l'ambiguïté de cet essor du film fixe ${ }^{27}$.

Ainsi, tout en reconnaissant "l'extrême richesse" de la production de films fixes, le congrès souligne que "nous n'étonnerons personne en disant que cette apparente richesse est souvent décevante. Faute de directives susceptibles d'éclairer les réalisateurs, ceux-ci travaillent quelques fois au hasard (plusieurs regrettent qu'une critique qualifiée ne guide leur effort). Trop de bandes sont pour le moins, médiocres. L'acheteuse, déçue, déplore les dépenses engagées et parfois se désintéresse d'un instrument pédagogique qui a, cependant, d'incontestables mérites ».

Mais le rapport final du congrès reste favorable à l'utilisation du film fixe, même dans les maternelles, en rappelant ses qualités :

"Le film fixe ne saurait être évincé par le film animé. Il a le mérite d'exister. Il en est d'utilisables, de bons, de très beaux. Le film fixe est de réalisation plus facile et moins onéreuse que la bande animée; sa technique de présentation est moins complexe et n'exige pas un équipement coûteux.

Les divers conférenciers ont exposé à maintes reprises, au congrès, les mérites d'une image déjà mobile et, de fait, plus vivante que celle de la gravure détachée ou de l'album.

De l'image fixe, elle a la valeur concrète. Un éclairage heureux peut mettre en valeur un certain aspect des choses qui en renouvelle la vision, peut la rendre plus éloquente pour l'observateur, plus étrange, plus touchante que la réalité elle-même.

Le film fixe est une image mobile et qui bénéficie auprès de l'enfant d'un peu du prestige du film 
animé. Elle déroule "l'histoire » dans une suite rythmée, avec ses surprises, ses temps d'arrêt, ses minutes d'émotion.

Elle a l'avantage sur l'image animée de permettre une lecture plus lente, des arrêts prolongés sur certaines visions, des retours en arrière au gré de l'enfant et de la maîtresse.

Détrôner le film fixe au profit du film animé? Nul n'y a songé. La bande fixe ouvre une riche carrière à celles qui veulent chercher, en dehors des thèmes tout trouvés. Les institutrices ont en elles toutes les ressources pour renouveler la production, pour inspirer heureusement les maisons d'édition.

Elles devront être exigeantes du point de vue de la qualité technique.

Beaucoup de firmes, d'amateurs, ont présenté leurs œuvres à Toulouse. Ils ont entendu éloges, critiques et vœux. D'anciennes maisons d'édition et de nouvelles sollicitent des directives, plusieurs sont décidées à consentir des nouvelles expériences, même coûteuses... Les conditions de travail sont donc excellentes ${ }^{28} »$ ?

Grâce à la circulaire de 1951, c'est «l'âge d'or » du film fixe, les maisons d'éditions se multiplient, elles sont plus d'une centaine, associées à une quarantaine de fabricants de projecteurs. Le nombre de films fixes réalisés en France des années 30 aux années 60 serait estimé de 15000 à $20000^{29}$ films différents.

Plus modestement, les films du Cedrhe se regroupent autour d'une vingtaine de maisons d'éditions ${ }^{30}$. Mais les films reflètent aussi les «dons» de grandes causes nationales comme la lutte contre l'alcoolisme et également des grands groupes privés qui voient dans le film fixe un moyen « d'entrer » dans l'école.

Les films ne possèdent pas toujours, voire très rarement, la notification de leurs auteurs. Néanmoins, pour les Éditions nouvelles pour l'enseignement, de nombreux films furent réalisés par Raymond Bettembos ${ }^{31}$, directeur pédagogique des Éditions nouvelles.

De par leurs thèmes et leurs objectifs, récréatifs, religieux ou didactiques, les films fixes ont été utilisés dans et hors les classes. Ils servent ainsi de support visuel au professeur lors de séquences d'enseignement pour de multiples niveaux avec une prépondérance pour les écoles maternelles et primaires.

\section{Objet de recherche pour une nouvelle « méthode de travail »?}

Créé en 1947, le laboratoire de psychopédagogie du centre audio-visuel de l'École normale supérieure de Saint-Cloud ${ }^{32}$ entreprend des expériences d'enseignement audio-visuel. Un article de 1949 relate une de ses expériences.

53 L'objectif déclaré de la recherche est de prouver l'intérêt de l'usage pédagogique du film et la volonté d'une « utilisation systématique dans les classes des aides visuelles d'enseignement ». "Encore convenait-il, au départ, de leur apporter la preuve que le rendement pédagogique d'une telle méthode de travail justifiait les frais à engager pour l'équipement des écoles et pour la formation d'un personnel expérimenté. C'est ce qui fit l'objet de notre expérience ${ }^{33}$ ».

54 À côté du film animé, du film sonore mais aussi des disques, de la radio ou de la télévision naissante mais néanmoins citée dans l'article, le film fixe présente pour les chercheurs des avantages indéniables :

«C'est le film fixe qui pourra répondre le mieux aux besoins de l'observation et aux possibilités matérielles, dans un autre cas, le film sera seul capable de rendre le mouvement qui restitue la vie. D'autre part, on peut pressentir une méthode qui, au 
lieu de juxtaposer à un enseignement de type traditionnel, oral et livresque pour l'essentiel, quelque vague complément audio-visuel, ferait, au contraire, du recours systématique à des aides le point de départ de toute étude et le centre même du travail scolaire. Ce sont ces idées neuves, sur des matériels déjà anciens, que nous voulons proposer à l'adhésion des maîtres français ».

Toutefois, il souligne des réserves importantes :

"L'utilisation des moyens audio-visuels ne doit en aucun cas se substituer à l'activité de l'élève ; ils ne peuvent remplacer l'expérimentation. Ils doivent susciter l'intérêt pour la recherche et non donner un vernis de connaissances ${ }^{36}{ }^{\prime}$.

62 L'introduction de ce nouveau médium, et derrière elle, la mise en activité des élèves, apparaît ambigüe car si l'espoir des pédagogues d'Éducation nouvelle est grand dans l'utilisation d'un nouveau moyen pédagogique, le risque est qu'il ne soit qu'un moyen certes plus moderne, peut-être plus efficace de poursuivre un enseignement traditionnel. 
Néanmoins, après un tel panégyrique, se pose la question des usages réels du film fixe dans les classes.

\section{Le film fixe, support d'une rénovation pédagogique : de l'espoir à la désillusion?}

65 En entrant dans les classes, le film fixe devient un enjeu entre les militants d'une rénovation pédagogique et les tenants d'un enseignement jugé « traditionnel ».

Mais c'est aussi pour certains chercheurs en "sciences de l'éducation » un objet d'étude et de recherche. En tout état de cause, le film fixe ne participe-t-il pas à une histoire des pratiques pédagogiques comme support privilégié des enseignants, avec le manuel scolaire, durant les années 50 et 60 ?

67 Car ce qui est récurrent en histoire de l'éducation, c'est qu'à chaque période, de nouveaux supports apparaissent, de "nouvelles technologies", qu'il s'agisse de projection sur verre, du film fixe et animé, de la télévision ou des outils numériques actuels. Ces outils pédagogiques provoquent à chaque fois des interrogations et des controverses sur leurs usages, leurs intérêts pédagogiques mais aussi sur leurs risques pour l'autorité de l'enseignant, pour les apprentissages et la transmission des savoirs.

68 Ainsi, il convient de s'interroger à la fois sur «l'accueil» de ce nouvel outil par les enseignants et de son appropriation didactique. Ensuite, au-delà des débats inhérents à l'introduction de ce nouvel outil, ses usages didactiques sont-ils nouveaux et innovants ou ne sont-ils qu'un vernis de modernité technique d'un enseignement traditionnel ? Audelà de cette vision manichéenne, le film fixe a-t-il influencé et infléchi les façons d'enseigner? Comme pour toute question d'histoire des pratiques, les sources nous manquent mais les revues professionnelles ainsi que les écrits des enseignants permettent d'esquisser plusieurs réflexions.

69 Les pédagogues de l'éducation nouvelle ont perçu dans le cinéma, un vecteur de modernité, d'innovation. Ovide Decroly dès 1906 utilise le film non du point de vue illustratif et éducatif mais comme outil de recherche et de diffusion de ses innovations pédagogiques.

Dès 1927, en créant la Cinémathèque coopérative de l'enseignement laïc, Célestin Freinet apparait comme l'un des plus novateurs sur la question en plaçant le cinéma comme un outil, au même titre que la correspondance scolaire, au service des apprentissages.

Dans son rapport intitulé « le cinéma, nouveau moyen d'expression » en 1961, Freinet est très clair :

« Le cinéma, et la photo dont il est issu, sont plus qu'un amusement ou même qu'un moyen d'éducation. Ils sont plus qu'un septième art. Ils sont une nouvelle forme de penser et de s'exprimer ${ }^{37} \%$.

72 Freinet place la question non seulement sur le plan pédagogique mais également sur le plan politique :

«Malheureusement toute technique nouvelle a son côté positif et son côté négatif. Et malheureusement aussi, dans le système social et économique actuel, les techniques nouvelles sont aux mains de grandes concentrations industrielles et financières, qui ne s'intéressent qu'à l'aspect bénéfique financier de l'opération et qui ne craignent pas de flatter les plus bas instincts de l'homme pour augmenter leurs profits ». 
Ensuite, il propose une réflexion sur la portée psychologique de l'image.

«Le règne de l'image, sous toutes ses formes, est en train de modifier dans des profondeurs et jusqu'en ses racines, le mode de pensée, d'expression et de vie de nos générations (...) L'image en général, l'image animée en particulier, sont en train de transformer les processus mentaux de nos enfants ».

74 Face à ce que Freinet nomme «l'hallucination » de l'image, il propose que les enseignants, non seulement utilisent le film mais réalisent eux-mêmes et construisent avec les enfants des scénarios. Ainsi, le film fixe en particulier n'est pas par essence innovant mais il peut l'être s'il devient un outil pédagogique d'éducation nouvelle. La question que pose Freinet reste donc: «comment se développer avec l'aide des outils nouveaux proposés par le monde moderne?».

\section{L'usage du film fixe dans les classes : une leçon modèle?}

Le film fixe, plus qu'un outil « innovant » par nature, reflète l'usage que l'on en fait. Quels indices possède-t-on? Comment retrouver l'archéologie des usages du film fixe?

Tout d'abord à travers les films de préparation. Par exemple, le film la Leçon avec projections cinématographiques ${ }^{38}$ est un film destiné à expliquer aux maîtres comment utiliser le cinéma dans la classe. Outre des informations concernant la maintenance et l'utilisation du projecteur, le film fournit une méthode de gestion de la leçon au moyen de l'outil cinématographique. Dans ce film, le maître pilote intégralement le dispositif de projection dans une salle de classe où un équipement fixe a été aménagé à cet effet. Le Cedrhe quant à lui, possède plusieurs notices explicatives rédigées par les éditeurs pour " aider » les enseignants à préparer leurs séances filmiques ${ }^{39}$.

Il s'agit tout au plus d'un cours dialogué où le film conforte ou illustre le discours du maître. Peut-on parler d'une étude documentaire? Les livrets d'accompagnement entendent en tout cas donner au maître les moyens d'une telle étude du point de vue du contenu. Mais très peu d'indications méthodologiques sur l'étude par plan ou autre sont données.

Eugène Reboul directeur de l'office de Saint-Étienne proposait une méthode qui favorise la mise en activité des élèves :

«Celle qui incorpore la projection à la leçon, qui l'accompagne pas à pas, et qui traduit immédiatement par l'image réelle l'explication plus ou moins abstraite du maître. La projection même prend place avec son titre sur le cahier de l'élève, titre accompagné d'une brève explication résumant les points essentiels, et suivant le cas, d'un petit croquis. (...) cette méthode présente divers avantages : elle évite la fatigue visuelle des élèves, car les arrêts du film sont volontairement fréquents ; par sa diversité, elle tient perpétuellement en éveil l'attention et l'intérêt; certains élèves ne sont plus tentés d'assister inertes et passifs à un cours que, sans le cinéma, ils auraient suivi distraitement. L'effort personnel est encouragé, accru, et la classe toute entière peut être tenue en haleine par des questions posées à propos et qui facilitent l'émulation ${ }^{40}$ ».

Dans les années cinquante et soixante, ce ne sont plus les « pionniers » adeptes du cinéma qui sont les fers de lance de l'accélération et de la diffusion du film fixe dans les classes mais bien l'institution scolaire qui y voit un "gage de modernité » et d'adaptation à la société. Les travaux du centre audio-visuel de Saint-Cloud légitiment « scientifiquement » 
cette vision. Le film fixe reste encore jusqu'au milieu des années soixante-dix l'outil audio-visuel le plus répandu dans les classes primaires. Même si à la fin des années soixante, la production de films fixes s'arrête, détrônée par les diapositives, le Cedrhe conserve de très nombreux films fixes achetés par les écoles communales du département de l'Hérault jusqu'en 1978.

Les pratiques de l'enseignement secondaire des années soixante évoluent avec la massification de la scolarisation et l'allongement de la scolarité obligatoire à 16 ans. Néanmoins, le cours magistral reste une pratique répandue et il faut attendre le milieu des années soixante-dix pour observer l'apparition généralisée des diapositives, du rétroprojecteur et de la télévision.

81 Pourtant la revue, Les bulletins de la radiotélévision scolaire, publiée au milieu des années soixante avait pour objectif d'accélérer et de généraliser l'usage de tous les moyens audiovisuels dans l'enseignement primaire et secondaire. En 1965, plusieurs articles tentent de proposer aux enseignants une pratique nouvelle de l'audio-visuel avec une « complémentarité entre cinéma, télévision et vues fixes ». Mais c'est finalement l'usage des nouveaux moyens audio-visuels qui est perçu comme novateur :

"L'image fixe ne pourrait-elle pas apporter également son appui aux émissions "compléments d'enseignement"? En langues vivantes, par exemple, où le document visuel limiterait la traduction dans la langue maternelle? La vue fixe, mobilisant l'attention, faciliterait la compréhension; elle aiderait à la mémorisation en développant des associations images-sons ${ }^{41} »$.

Mais, la revue pose régulièrement la question de savoir si le matériel audio-visuel est un moyen efficace d'éducation ${ }^{42}:$ "dans quelle mesure peut-on compenser la fluidité et l'irréversibilité de la télévision en faisant appel à la projection fixe au cours de l'exploitation ${ }^{43}$ ?».

83 Ensuite, plus intéressant pour nous, sur la question de l'impact réel du film fixe dans les classes, la revue titre en 1964 : «les maîtres dressent un premier constat ». Ce premier bilan tente d'analyser l'usage de l'audio-visuel dans les classes.

La circulaire du ministère de l'Éducation nationale du 14 juin 1963 désigna en effet une série d'établissements pilotes chargés d'utiliser de façon expérimentale tous les moyens audio-visuels. Les rapports transmis par les établissements concernent donc l'ensemble des moyens audiovisuels. Très clairement, de nombreux rapports soulignent le fait que beaucoup d'enseignants s'inquiètent d'être " dépossédés » de leur rôle par ces nouveaux outils. Ensuite, un clivage générationnel est aussi perceptible entre les jeunes enseignants qui seraient ainsi plus prêts à utiliser de nouveaux procédés et les enseignants de plus longue date moins familiarisés avec les nouveaux médias.

Toutefois, au-delà de la motivation de l'image en tant que telle, réelle ou supposée, il semble bien que l'usage généralisé du film fixe soit issu d'une réelle ambiguïté. Le film fixe est perçu « moderne » comme la diapositive qui le supplante à partir des années 60 mais son usage reste avant tout illustratif. Ensuite, il ne « contredit » pas le maître, il est un auxiliaire docile. Enfin, il peut parfois « domestiquer » le maître en imposant la suite logique de ses vues fixes, en proposant une interprétation comme pourrait le faire d'ailleurs le manuel scolaire.

Ainsi l'histoire du film fixe apparaît bien comme un outil pédagogique au service de l'histoire de l'éducation en permettant d'esquisser une histoire des pratiques pédagogiques mais aussi de l'Éducation nouvelle. En effet, le film fixe fut perçu à son 
origine comme un outil « innovant » au service d'une nouvelle pédagogie, mais sans réelle transformation de l'organisation scolaire et pédagogique, il ne sera qu'un outil au service d'un enseignement « traditionnel » fondé sur la seule transmission des savoirs.

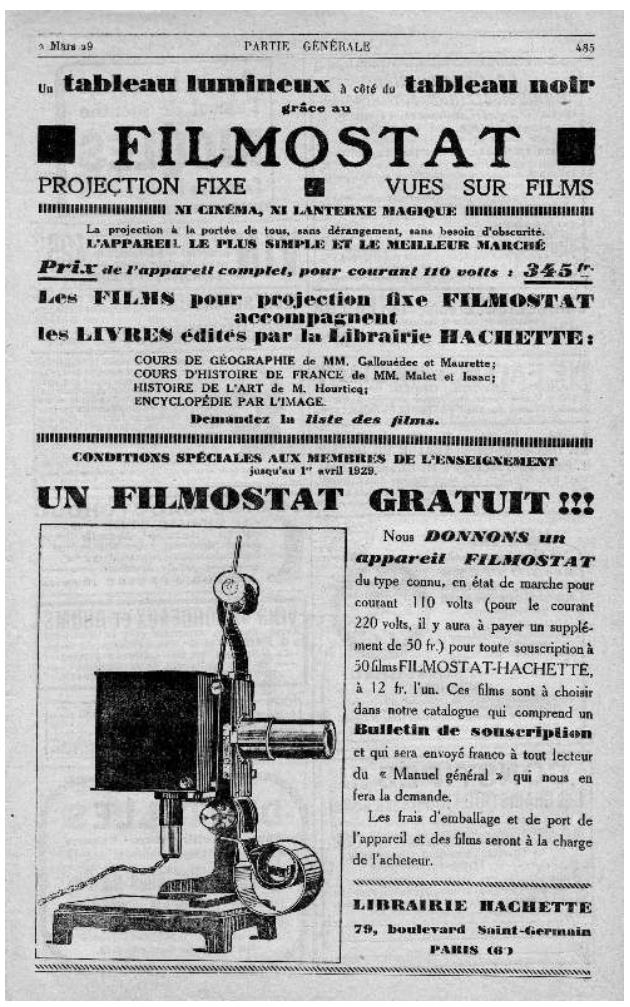

Publicité Filmostat, Manuel général de l'instruction primaire, journal hebdomadaire des instituteurs et institutrices, 96e année, №23, 2 mars 1929, p. 485.

\section{NOTES}

1. Une première version de cet article a été publiée dans la revue le Cartable de Clio. Wagnon, S. et André, H. (2013). Le film fixe : un outil pédagogique au service de l'Histoire de l'Éducation. Le cartable de Clio, $\mathrm{n}^{\circ} 13$.

2. Guibbert, P. et Oms, M. (1993). Comment le cinéma français a-t-il raconté l'histoire de France? Paris : CNDP.

3. Guibbert, P. (1972). Circonstances, modalités et significations de l'œuvre d'Eisenstein. T.1: Aspects et perspectives (littérature comparée), T.2: Dossiers filmographiques, Bibliographie, Annexes.

4. Guibbert, P. (1992). Le film fixe, vecteur d'une pédagogie impure, Tréma, n², pp. 79-82.

5. Vignaux, V. (2007). Jean Benoit-Lévy ou le corps comme utopie, une histoire du cinéma éducateur dans l'entre-deux-guerres en France. Paris : AFRHC, $254 \mathrm{p}$.

6. Coissac, G.-M. (1926). Le cinématographe et l'enseignement : nouveau guide pratique approuvé et adopté par le ministère de l'instruction publique. Paris : Larousse : Éditions du cinéopse.

7. Nourrisson, D. et Jeunet, P. (dir.) (2011). Cinéma-École : aller-retour. Saint-Étienne : Presses Universitaires de Saint-Étienne. 
8. Lantenois, A. (1933). Ce que l'instituteur doit savoir. Paris : Nathan.

9. Vignaux, V. (2009). Les animateurs français et le Pathé-Baby ou des usages privés des images cinématographiques dans la France de l'entre-deux-guerres. Mille huit cent quatre-vingt-quinze, $\mathrm{n}^{\circ} 59$, no 3, pp.82-95.

10. Dujonc, I. (2005). Les films fixes et les missionnaires de l'Ouest de la France. Annales de Bretagne et des pays de l'Ouest, $n^{\circ} 2$; Bonafoux, C. (2012). Les catholiques français devant le cinéma entre désir et impuissance, Essai d'une histoire du public catholique. Cahiers d'études du religieux. Recherches interdisciplinaires, Numéro spécial.

11. Coissac, G.-M. (1926). Le cinématographe et l'enseignement: nouveau guide pratique approuvé et adopté par le ministère de l'instruction publique. Paris : Larousse : Éditions du cinéopse.

12. Pautrier, C. (1904). Comment lutter, comment agir ! Le fascinateur, avril, p.101.

13. Thomas, F. (1899). L’enseignement visuel. La revue pédagogique, $n^{\circ} 12$, décembre, p.482.

14. Bessou, A. (1920). Rapport de la Commission extraparlementaire chargée d'étudier les moyens de généraliser l'application du cinématographe dans les différentes branches de l'enseignement. Paris : Imprimerie nationale.

15. Bessou, A. (1920). L'emploi du cinématographe dans les différentes branches de l'enseignement. La revue pédagogique, février, p.128.

16. Ciné schola, bulletin de la ligue pour l'enseignement par la cinématographie, $\mathrm{n}^{\circ} 1$, novembre 1922.

17. Laborderie, P. (2012). Les Offices du cinéma scolaire et éducateur à l'épreuve des publics. Conserveries mémorielles, $\mathrm{n}^{\circ} 12$.

18. Borde, R. et Perrin, C. (1992). Les offices du cinéma éducateur et la survivance du muet (1825-1940). Lyon : PUL.

19. Vignaux, V. (2007). Jean Benoit-Lévy ou le corps comme utopie, une histoire du cinéma éducateur dans l'entre-deux-guerres en France. Paris : AFRHC.

20. Lefebvre, T. (2001). Films fixes et santé publique. Revue d'histoire de la pharmacie, $\mathrm{n}^{\circ} 331$, pp. 381-399 ; Freyssinet-Dominjon, J., Nourrisson, D. (2008). L'école face à l'alcool. L'enseignement de l'antialcoolisme en France 1870-1970, Saint-Étienne: Publications de l'Université de SaintÉtienne, coll. IUFM.

21. Tout cinéma avril 1930

22. Nourrisson, D. et Jeunet, P. (dir.). (2011). Cinéma-École : aller-retour. Saint-Étienne : Presses Universitaires de Saint-Étienne.

23. Bulletin Officiel de l'Education Nationale $n^{\circ} 6$ du 7 février 1952 et Instruction du 30 janvier 1952 modifiée par la circulaire du 21 février 1952: Production et acquisition des films d'enseignement par le ministère de l'éducation nationale ; acquisition des films d'enseignement par les établissements scolaires ; régime de distribution des films par les cinémathèques de prêt (RLR 177-2a).

24. Ibid.

25. Nourrisson, D. et Jeunet, P, (dir.), Cinéma-École : aller-retour, Saint-Etienne : Presses Universitaires de Saint-Étienne, 2011.

26. Fonds films fixes du Cedrhe (Centre d'Études, de Documentation et de Recherches en Histoire de l'Éducation).

27. Thomet, M. \& Hebraud, Y. (1952). Le film au service de la première éducation, Les cahiers de pédagogie moderne pour l'enseignement du premier degré. Paris : éditions Bourrelier 1952.

28. Ibid.

29. Lefebvre, T. (2001). Films fixes et santé publique. Revue d'histoire de la pharmacie, n³31, pp. 381-399.

30. Les maisons d'éditions les plus présentes sont en particulier pour les films religieux, Bloud \& Gay, Maison de la Bonne Presse, Éditions du Berger, La Centrale Saint Jean, Le Cinéma dans les 
œuvres et pour les films scolaires, Éditions de la Photoscopie, Filmostat, Fixus-films, Larousse, Les Beaux Films, Les Éditions Filmées, Les Éditions Nouvelles pour l'Enseignement, l'Office de Documentation par le Film.

31. En 1950, Raymond Bettembos signe l'introduction d'une collection de films fixes intitulé De la Révolution à nos jours, Paris, Éditions nouvelles pour l'enseignement; voir Amalvi, C. (2001). Répertoire des auteurs de manuels scolaires et de livres de vulgarisation historique de langue française de 1660 à 1960. Paris : La Boutique de l'histoire, p. 37.

32. Leboutet, L. (1949). Une expérience d'enseignement audiovisuel. Enfance, tome $2, \mathrm{n}^{\circ} 1, \mathrm{pp}$. 77-81.

33. Leboutet, L. (1949). Une expéreince d'enseignement audiovisuel. Enfance, tome 2, nº et extraits suivants.

34. Lafon, R. (1963). Vocabulaire de psychopédagogie et de psychiatrie de l'enfant. Paris : PUF.

35. Ibid. Seconde édition de 1969, p.75.

36. Ibid.

37. Freinet, $\mathrm{C}$. le cinéma, nouveau moyen d'expression, Rencontres internationales du film pour la jeunesse, Cannes 12-18 juin 1961.

38. Vignaux, V. (2009). Les animateurs français et le Pathé-Baby ou des usages privés des images cinématographiques dans la France de l'entre-deux-guerres. Mille huit cent quatre-vingt-quinze, $\mathrm{n}^{\circ}$ 59, no 3, pp.82-95.

39. Fonds film fixe, Cedrhe. Cf. «Achetez votre film comme vous achetez un livre » des éditions Éditafilms ; Les préparations de classe par le film des Éditions nouvelles pour l'enseignement.

40. Reboul, E. (1926). Le cinéma scolaire et éducateur. Paris : PUF, p. 23.

41. Minéo, S. (1965). La radiovision scolaire. Bulletin de la radio-télévision scolaire, $n^{\circ} 34$, p.14.

42. Ibid. p.24.

43. Bulletin de la radio-télévision scolaire, $n^{\circ} 11,22$ juin 1964.

\section{ABSTRACTS}

Filmstrips was, with the textbook, one of the most used educational tools in France from the 30s to the 60s. Forgotten by the History of the contemporary education, it was from the beginning of the 20s, an ideological stake between the catholic school and the secular school, but also an educational stake. Patrimonial object since the arrival of the slide in the 60 s and the computer from the 80s, the history of the filmstrips analysing, by its confined chronological limits, offers the possibility of studying a didactic object and of participating in the construction of this material history of education. Our contribution wishes to bring to light on one hand the characteristics of the filmstrips and its practices as indications of the history of educational practices and on the other hand, to analyze this history in the context of the evolution of education policies.

Le film fixe fut, avec le manuel scolaire, l'un des outils pédagogiques les plus utilisés dans la France des années 30 aux années 60. Néanmoins, grand oublié de l'Histoire de l'éducation contemporaine, il fut pourtant, dès sa naissance au début des années 20 , un enjeu idéologique entre l'école catholique et l'école laïque, mais aussi un enjeu pédagogique. Objet patrimonial depuis l'arrivée de la diapositive dans les années 60 et des supports informatiques à partir des années 80 , l'histoire du film fixe, par ses limites chronologiques circonscrites, offre la possibilité 
d'étudier un objet didactique et de participer à la construction de cette histoire matérielle de l'éducation. Notre contribution souhaite mettre en lumière d'une part les caractéristiques du film fixe et ses usages comme indices de l'histoire des pratiques pédagogiques et d'autre part, analyser cette histoire dans le contexte de l'évolution des politiques éducatives.

INDEX

Mots-clés: film fixe, histoire de l'éducation

Keywords: education history, filmstrips

\section{AUTHORS}

\section{SYLVAIN WAGNON}

Faculté d'Éducation - Université de Montpellier 2 LIRDEF

\section{HÉLÈNE ANDRÉ}

Cedrhe - Faculté d'Éducation - Université de Montpellier 2(Centre d'études, de documentation et de recherches en histoire de l'éducation) 